International Journal of Engineering \& Technology, 7 (3.10) (2018) 98-102
International Journal of Engineering \& Technology
SPC
Website: www.sciencepubco.com/index.php/IJET
Research paper

\title{
Seismic Response and Analysis of RCC Block Shear Wall in Assymetric Building Using ETABS
}

\author{
T.Subramani $^{1^{*}}$, D.Ananthi ${ }^{2}$ \\ $1^{*}$ Managing Director, Priyanka Associates (Civil Engineers and Valuers), Salem, TamilNadu, India. \\ ${ }^{2}$ Assistant Engineer, RD, Namakkal., TamilNadu, India. \\ *Corresponding author E-mail: tsmcivil2007@gmail.com
}

\begin{abstract}
Shear wall is the structural member which with stand the horizontal or lateral forces. The structures are subjected to dynamic loading which have an effect on in general. Our proposed plan is to save you the structural deformation because of dynamic loading. While partitions are situated in tremendous positions in a constructing, they may be very efficient in resisting lateral masses ori ginating from wind or earthquakes. This paper provides designated analyses of experimental and analytical has accomplished end result has generated. Our undertaking focuses on reading the impact of twist, as a system-stage impact, on the displacement and strength demands of the building's separate seismic force resisting system (SFRS) wall components. The look at evaluates the individual wall contributions to the overall building response characteristics within both the elastic and the inelastic reaction phases.
\end{abstract}

Keywords: Seismic response, Analysis, RCC Block, Shear wall, Assymetric, ETABS

\section{Introduction}

Commonly a concrete frame is layout to face up to gravity load and made stiff by using providing brittle masonry filler wall within frame, therefore there may be greater opportunity that because of its extra stiffness, it'll entice more of the earthquake forces and fail in shear because of failure of brittle masonry. Consequently creation of shear wall in constructing which comes below seismic region avoids fall apart.

By using enhancing ductility and electricity dissipation ability within the structure, the caused seismic forces may be decreased, and a more reasonably priced shape acquired, or as an alternative, the chance of collapse reduced. Hence constructing with lateral load resisting machine comprising a twin device inclusive of ductile moment resisting space body and ductile flexural (shear) wall qualify for terribly low seismic caused forces.

They're typically used inside the structures to withstand the outcomes of gravity hundreds and storey shears. Shear walls are vertical elements inside the lateral force resisting system that transmit lateral forces from the diaphragm above to the diaphragm underneath or to the muse. Shear walls might also bearing partitions inside the gravity load system or they'll be additives in dual gadget framed a good way to resist most effective lateral masses.

\section{Methodology}

Fig. 1 shows the methodology of the study

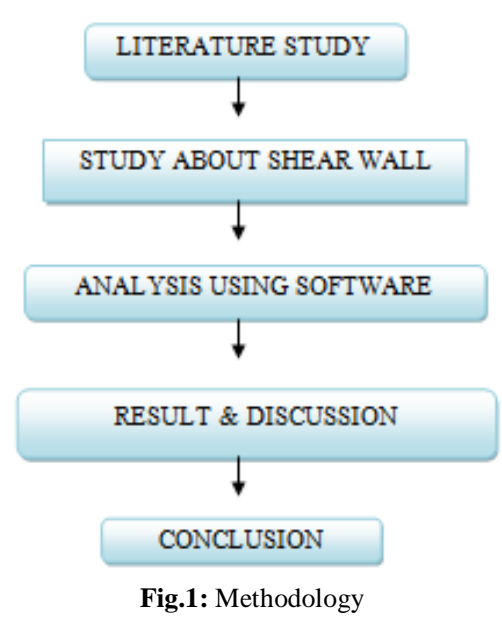

\section{Shear Wall}

Shear walls are vertical components of the horizontal pressure resisting gadget. They may be commonly wooden frame stud partitions included with a structural sheathing fabric like plywood. While the sheathing is nicely fixed to the stud wall framing, the shear wall can face up to forces directed alongside the length of the wall. When shear walls are designed and constructed nicely, they'll have the power and stiffness to withstand the horizontal forces. Fig. 2 indicates the typical shear wall. 


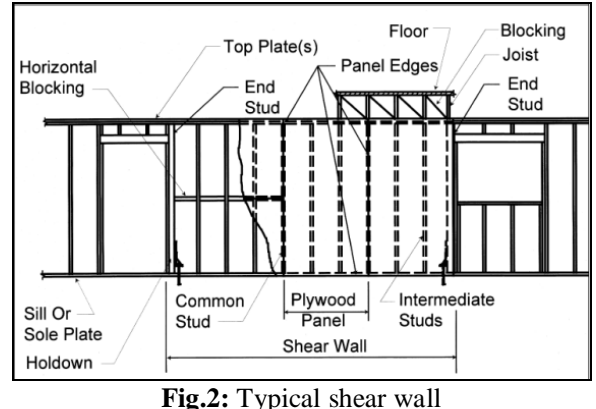

Fig.2: Typical shear wall

Fig. 3 shows the rectangular structures.

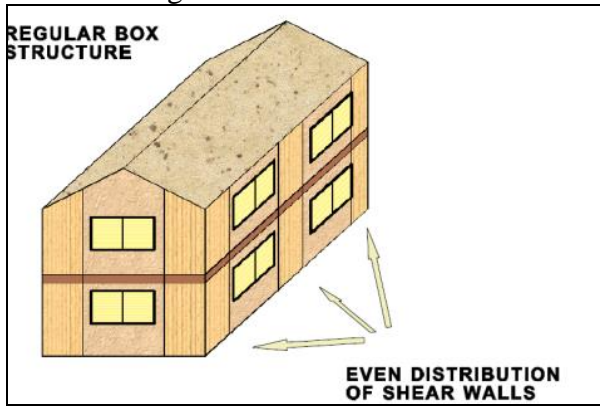

Fig.3: Rectangular structure

That's the reason why most retrofit work uses walls with continuous footings underneath them as shear walls. Fig. 4 shows the horizontal alignment of cripple walls

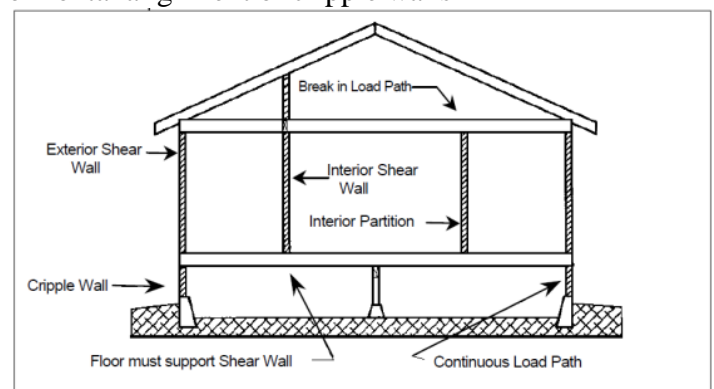

Fig.4: Horizontal alignment of cripple walls

Fig. 5 shows the Vertical offset of shear walls

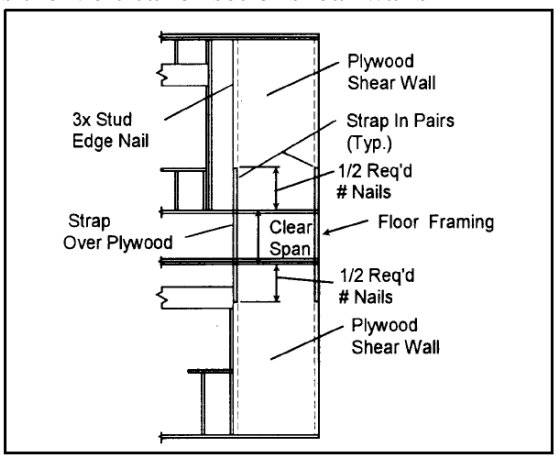

Fig.5: Vertical offset of shear walls

Another sort of alignment problem happens whilst the ends of shear walls do now not align from tale to tale. This situation creates the need for additional framing members and connections in the walls for hold down devices. Holdown gadgets ought to switch the uplift from the shear wall to framing contributors which can withstand it. Whilst complete top studs aren't available, unique connections have to be delivered. These connections indicate duty or correctness to gather sufficient of the structure's framing to face up to the uplift.

\section{Earthquake}

Earthquakes have many different outcomes except vibrating the systems in response to floor shaking at its basis. These different results may even exceed that because of vibration. Unfortunately, the technique in their estimation and the desired steps for the layout are considered outdoor the scope of structural engineering. Distinctive seismic resistant layout codes have provisions to recollect the vibration of structures. However, these codes do not have any provision to take care of other results. but, structural engineers should be aware of the intensity of the dangers so that you can taking precautionary measures both within the layout of systems, advising customers in selecting right websites in such zones or making them aware about the significance of right preservation of the systems and other considerations the clients need to observe up at the same time as using the designed structures. The different direct and indirect effects of earthquakes are mentioned in the following section.

1. Direct Seismic Effects

2. Indirect Seismic Effects

\section{Software}

The modern version of ETABS continues in that subculture, incorporating structural detail terminology this is used on a day by day basis (columns, beams, bracings, shear partitions etc.), opposite to the commonplace civil engineering programs that use terms along with nodes, contributors etc. additionally, it offers many computerized features for the formation, evaluation and design of the structural gadget in an green, fast and smooth manner. Evaluation and design of building systems with a structural device which includes beams, slabs, columns, shear walls and bracings. Distinct substances can be assigned to the structural factors within the identical version inclusive of steel, $\mathrm{RC}$, composite or some other user-defined cloth. Easy and automated era of gravity and lateral masses (seismic and wind hundreds) while as compared with other FE standard evaluation packages.

\section{Analysis Results}

Fig.6 shows the ETABS modeling

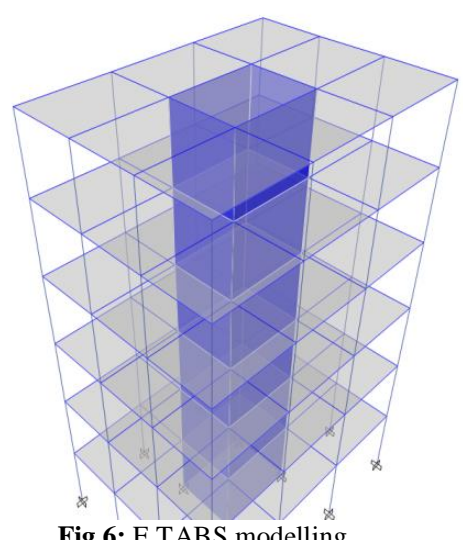

Fig.6: E TABS modelling

\subsection{Structure Data}

This area provides model geometry information, including items such as story levels, point coordinates, and element connectivity. 


\subsubsection{Storey Data}

Table 1 shows the storey data

Table 1: Storey data

\begin{tabular}{|c|c|c|c|c|c|}
\hline Name & $\begin{array}{c}\text { Height } \\
\text { mm }\end{array}$ & $\begin{array}{c}\text { Elevation } \\
\text { mm }\end{array}$ & $\begin{array}{c}\text { Master } \\
\text { Story }\end{array}$ & $\begin{array}{c}\text { Similar } \\
\text { To }\end{array}$ & $\begin{array}{c}\text { Splice } \\
\text { Story }\end{array}$ \\
\hline Story6 & 3500 & 21000 & Yes & None & No \\
\hline Story5 & 3500 & 17500 & No & Story6 & No \\
\hline Story4 & 3500 & 14000 & No & Story6 & No \\
\hline Story3 & 3500 & 10500 & No & Story6 & No \\
\hline Story2 & 3500 & 7000 & No & Story6 & No \\
\hline Story1 & 3500 & 3500 & No & Story6 & No \\
\hline Base & 0 & 0 & No & None & No \\
\hline
\end{tabular}

\subsection{Loads}

This area provides loading information as applied to the model.

\subsubsection{Load Patterns}

Table 2 shows the load patterns

Table 2: Load patterns

\begin{tabular}{|c|c|c|c|}
\hline Name & Type & $\begin{array}{c}\text { Self-Weight } \\
\text { Multiplier }\end{array}$ & Auto Load \\
\hline Dead & Dead & 1 & \\
\hline Live & Live & 0 & \\
\hline Seismic & Seismic & 0 & IS18932002 \\
\hline
\end{tabular}

\subsubsection{Load Cases}

Table 3 shows the load cases

Table 3: Load Cases - Summary

\begin{tabular}{|c|c|}
\hline Name & Type \\
\hline Dead & Linear Static \\
\hline Live & Linear Static \\
\hline Seismic & Linear Static \\
\hline
\end{tabular}

\subsection{Analysis Results}

This area provides analysis results.

\subsubsection{Structure Results}

Table 4 shows the base reactions.

Table 4: Base Reactions

\begin{tabular}{|c|c|c|c|c|c|c|}
\hline Load & $\mathbf{F X}$ & $\overline{F Y}$ & $\overline{F Z}$ & $\mathrm{Mx}$ & $\mathbf{M Y}$ & $\mathbf{M Z}$ \\
\hline Case Combo & $\mathrm{bN}$ & $\mathrm{kNx}$ & $b x$ & bNI-m & $\mathrm{bN}-\mathrm{m}$ & $\mathrm{k}: \mathbb{N}-\mathrm{m}$ \\
\hline Dead & 0 & 0 & 9588.1004 & 43146.452 & -57528.6027 & 0 \\
\hline Live & 0 & 0 & 0 & 0 & 0 & 0 \\
\hline Seismic 1 & -825.6126 & 0 & 0 & 0 & -13622.2722 & 37152565 \\
\hline Seismic 2 & 0 & -825.6126 & 0 & 13622.2722 & 0 & -4953.6753 \\
\hline Seismic 3 & -825.6126 & 0 & 0 & 0 & -13622.2722 & 4086.7822 \\
\hline Seismic 4 & 0 & -826.6126 & 0 & 13622.2722 & 0 & -5449.0429 \\
\hline Seismic 5 & -825.6126 & 0 & 0 & 0 & -13622.2722 & 3343.7309 \\
\hline Seismic 6 & 0 & -825.6126 & 0 & 13622.2722 & 0 & -4458.3078 \\
\hline DWall & 0 & 0 & 14382.1507 & 64719.678 & -86292.904 & 0 \\
\hline DWa12 & 0 & 0 & 14382.1507 & 64719.678 & $-\$ 6292904$ & 0 \\
\hline DWal3 Max & 0 & 0 & 11505.7205 & 68122.469 & -690343232 & 4904.1386 \\
\hline DWal3 Min & \begin{tabular}{|l|l|}
-990.7351 \\
\end{tabular} & \begin{tabular}{|l|l|}
-990.7351 \\
\end{tabular} & 11505.7205 & 51776.7424 & -85381.0498 & -6538.8515 \\
\hline DWal4 Max & 990.7351 & 990.7351 & 11505.7205 & 51775.7424 & -52687.5966 & 6538.8515 \\
\hline DWal4 Min & 0 & 0 & 11505.7205 & 35429.0158 & -69034.3232 & -4904.1386 \\
\hline DWal5 Max & 0 & 0 & 14382.1507 & 85153.0863 & -86292904 & 6130.1732 \\
\hline DWa15 Min & -1238.4188 & -1238.4188 & 14382.1507 & 64719.678 & -106726 & -8173.5643 \\
\hline DWal6 Max & 1238.4188 & 1238.4188 & 14382.1507 & 64719.678 & -65859.4958 & 8173.5643 \\
\hline DWal6 Min & 0 & 0 & 14382.1507 & 44286.2698 & -86292.904 & -6130.1732 \\
\hline DWal7 Max & 0 & 0 & 86292904 & 59265.2151 & -51775.7424 & 6130.1732 \\
\hline DWal7 Min & $-1238.418 s$ & $-1238.418 s$ & 86292904 & 38831.8068 & -72209.1507 & -8173.5643 \\
\hline DWals Max & 1238.4188 & 1238.4188 & 86292904 & 38831.8068 & -31342.3342 & 8173.5643 \\
\hline Min & 0 & $0_{0}$ & 86292904 & 18398.3986 & -51775.7424 & 30 \\
\hline
\end{tabular}

Table 5 shows the storey stiffness.

Table 5: Storey stiffness

\begin{tabular}{|c|c|c|c|c|c|c|c|}
\hline Story & $\begin{array}{l}\text { Load } \\
\text { Case }\end{array}$ & $\underset{k D \mathbb{N}}{\text { Shear } \mathbf{x}}$ & $\underset{\substack{\text { Drift Xm } \\
\text { mm }}}{ }$ & $\begin{array}{c}\text { Stiffness } \\
\mathbf{X} \\
\mathbf{X} / \mathbf{m}\end{array}$ & $\underset{k N X}{S h e a r ~ Y ~}$ & $\begin{array}{c}\text { Drift Y } \\
\text { mand }\end{array}$ & $\begin{array}{c}\text { Stiffness } \\
\mathbf{Y} \\
\mathbf{K} \mathbf{N} / \mathbf{m}\end{array}$ \\
\hline Story6 & Seismic 1 & 269.5376 & 0.6 & 421447.6 & 0 & 0 & 0 \\
\hline Storys & Seismic 1 & 522.299 & 0.7 & 775743.6 & 0 & o & o \\
\hline Story 4 & Seismic 1 & 684.0662 & 0.7 & 1038632 & 0 & 0 & o \\
\hline Story 3 & Seismic 1 & 775.0603 & 0.6 & 1320042 & 0 & 0 & 0 \\
\hline Story2 & Seismic 1 & 815.5021 & 0.4 & $\begin{array}{l}1812809 . \\
181287 .\end{array}$ & o & 0 & 0 \\
\hline Story 1 & Seismic 1 & 825.6126 & 0.2 & 3596231. & o & 0 & 0 \\
\hline Story-6 & Seismic 2 & 0 & 0 & 0 & 269.5376 & 0.8 & 33 \\
\hline Storys & Seismic 2 & 0 & 0 & 0 & 522.299 & 0.9 & \\
\hline Story 4 & Seismic 2 & o & - & 0 & 684.0662 & 0.9 & \\
\hline Story3 & Seismic 2 & o & 0 & 0 & 775.0603 & 0.8 & \\
\hline Story 2 & Seismic 2 & 0 & 0 & 0 & 815.5021 & 0.6 & 3594 \\
\hline Story 1 & Seismic 2 & 0 & o & 0 & 825.6126 & 0.3 & 26988 \\
\hline Story6 & Seismic 3 & 269.5376 & 0.6 & 421447.6 & 0 & 0.01196 & o \\
\hline Storys & Seismic 3 & 522.299 & 0.7 & $\frac{31}{775743.6}$ & 0 & 0.02143 & 0 \\
\hline Story 4 & Seismic 3 & 684.0662 & 0.7 & 1038632 & 0 & 0.02827 & \% \\
\hline Story 3 & Seismic 3 & 775.0603 & 0.6 & $\begin{array}{l}1320042 \\
150\end{array}$ & o & 0.0321 & o \\
\hline Story 2 & Seismic 3 & 815.5021 & 0.4 & 1812809 & 0 & 0.03405 & o \\
\hline Story1 & Seismic 3 & 825.6126 & 0.2 & 3596231 & o & 0.02678 & 0 \\
\hline Story 6 & Seismic 4 & 0 & 0.01196 & & 269.5376 & 0.8 & 331851 \\
\hline Storys & Seismic 4 & 0 & 0.02143 & 0 & 522.299 & 0.9 & 602212 \\
\hline Story 4 & Seismic 4 & o & 0.02827 & $\circ$ & 684.0662 & 0.9 & 795591.0 \\
\hline Story 3 & Seismic 4 & o & 0.0321 & 0 & 775.0603 & 0.8 & 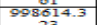 \\
\hline Story $2_{2}$ & Seismic 4 & - & 0.03405 & - & 815.5021 & 0.6 & 135941 \\
\hline Story 1 & Seismic 4 & o & 0.02678 & $\circ$ & 825.6126 & 0.3 & $\begin{array}{l}269885 \\
26985\end{array}$ \\
\hline Storys 6 & Seismic S & 269.5376 & 0.6 & 421447.6 & o & 0.01196 & 78 \\
\hline Storys & Seismic S & 522.299 & 0.7 & 775743.6 & $\circ$ & 0.02143 & o \\
\hline Story 4 & Seismic S & 684.0662 & 0.7 & 1038632 & o & 0.02827 & - \\
\hline Story 3 & Seismic s & 775.0603 & 0.6 & 1320042 & 0 & 0.0321 & 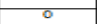 \\
\hline Story 2 & Seismic S & 815.5021 & 0.4 & 1812809. & $\circ$ & 0.03405 & \% \\
\hline Story 1 & Seismic S & 825.6126 & 0.2 & $\begin{array}{l}35967 \\
3545 \\
455\end{array}$ & o & 0.02678 & - \\
\hline Storys & Seismic o & o & 0.01196 & 8 & 269.5376 & 0.8 & 851.5 \\
\hline Storys & Seismic 6 & o & 0.02143 & - & 522.299 & 0.9 & \\
\hline Story 4 & Seismic o & o & 0.02827 & - & 684.0662 & 0.9 & \\
\hline Story 3 & Seismic of & $\circ$ & 0.0321 & $\circ$ & 775.0603 & 0.8 & 514 \\
\hline Story 2 & Seismic of & 0 & 0.03405 & 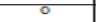 & 815.5021 & 0.6 & \\
\hline Story 1 & Seismic of & 0 & 0.02678 & & 825.6126 & 0.3 & $\frac{354}{69885}$ \\
\hline
\end{tabular}

\subsubsection{Modal Results}

Table 6 shows the modal periods and frequencies.

Table 6: Modal Periods and Frequencies

\begin{tabular}{|l|c|c|c|c|c|}
\hline Case & Mode & $\begin{array}{c}\text { Period } \\
\text { sec }\end{array}$ & $\begin{array}{c}\text { Frequency } \\
\text { sxc/sec }\end{array}$ & $\begin{array}{c}\text { Circular } \\
\text { Frequency } \\
\text { rad/sec }\end{array}$ & $\begin{array}{c}\text { Eigemvalse } \\
\text { rad }^{2} / \text { sec }^{2}\end{array}$ \\
\hline Modal & 1 & 0.294 & 3.397 & 21.3429 & 455.5191 \\
\hline Modal & 2 & 0.257 & 3.893 & 24.4618 & 598.3781 \\
\hline Modal & 3 & 0.219 & 4.566 & 28.6862 & 822.8962 \\
\hline Modal & 4 & 0.073 & 13.668 & 85.8793 & 7375.2591 \\
\hline Modal & 5 & 0.073 & 13.71 & 86.142 & 7420.4398 \\
\hline Modal & 6 & 0.062 & 16.001 & 100.54 & 10108.2905 \\
\hline Modal & 7 & 0.044 & 22.642 & 142.2625 & 20238.6238 \\
\hline Modal & 8 & 0.035 & 28.513 & 179.1543 & 32096.2809 \\
\hline Modal & 9 & 0.032 & 31.257 & 196.396 & 38571.3898 \\
\hline Modal & 10 & 0.03 & 33.156 & 208.3274 & 43400.295 \\
\hline Modal & 11 & 0.026 & 39.118 & 245.7835 & 60409.5107 \\
\hline Modal & 12 & 0.024 & 42.508 & 267.0844 & 71334.0915 \\
\hline
\end{tabular}

Table 7 shows the modal participating mass ratios (Part 1 of 2)

Table 7: Modal participating mass ratios (part 1 of 2)

\begin{tabular}{|l|c|c|c|c|c|c|c|c|}
\hline Case & Mode & $\begin{array}{c}\text { Period } \\
\text { sec }\end{array}$ & UX & UY & UZ & $\begin{array}{c}\text { Sum } \\
\text { UX }\end{array}$ & $\begin{array}{c}\text { Sum } \\
\text { UY }\end{array}$ & $\begin{array}{c}\text { Sum } \\
\text { UZ }\end{array}$ \\
\hline Modal & 1 & 0.294 & 0 & 0.72 & 0 & 0 & 0.72 & 0 \\
\hline Modal & 2 & 0.257 & 0.7155 & 0 & 0 & 0.7155 & 0.72 & 0 \\
\hline Modal & 3 & 0.219 & 0 & 0 & 0 & 0.7155 & 0.72 & 0 \\
\hline Modal & 4 & 0.073 & 0 & 0 & 0 & 0.7155 & 0.72 & 0 \\
\hline Modal & 5 & 0.073 & 0 & 0.1949 & 0 & 0.7155 & 0.9148 & 0 \\
\hline Modal & 6 & 0.062 & 0.2022 & 0 & 0 & 0.9177 & 0.9148 & 0 \\
\hline Modal & 7 & 0.044 & 0 & 0 & 0 & 0.9177 & 0.9148 & 0 \\
\hline Modal & 8 & 0.035 & 0 & 0.056 & 0 & 0.9177 & 0.9708 & 0 \\
\hline Modal & 9 & 0.032 & 0 & 0 & 0 & 0.9177 & 0.9708 & 0 \\
\hline Modal & 10 & 0.03 & 0.0542 & 0 & 0 & 0.9719 & 0.9708 & 0 \\
\hline Modal & 11 & 0.026 & 0 & 0 & 0 & 0.9719 & 0.9708 & 0 \\
\hline
\end{tabular}

Fig.7 shows the stress diagram 


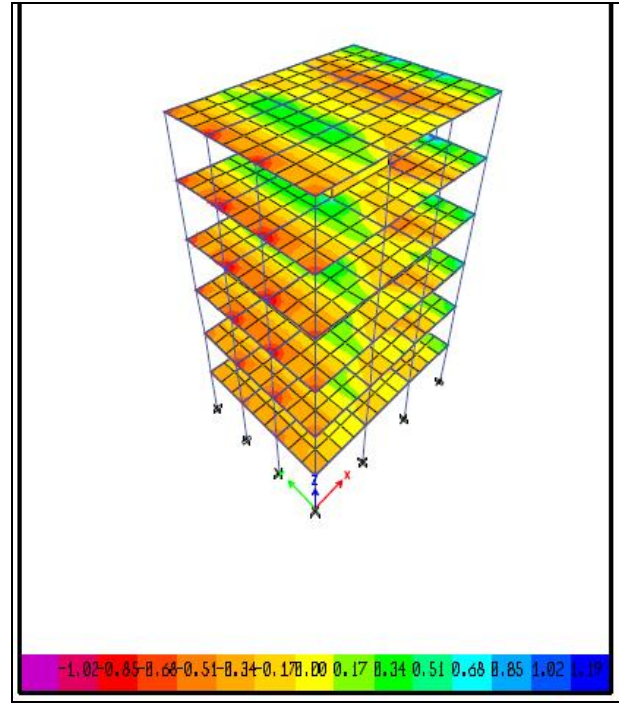

Fig.7: Stress diagram

\subsubsection{Etabs 2015 Shear Wall Design}

Table 8 shows the Pier details.

Table 8: Pier details

\begin{tabular}{c|c|c|c|c|c|c}
\hline $\begin{array}{c}\text { Story } \\
\text { ID }\end{array}$ & $\begin{array}{c}\text { Pier } \\
\text { ID }\end{array}$ & $\begin{array}{c}\text { Centroid X } \\
(\mathbf{m m})\end{array}$ & $\begin{array}{c}\text { Centroid Y } \\
(\mathbf{m m})\end{array}$ & $\begin{array}{c}\text { Length } \\
\mathbf{( m m )}\end{array}$ & $\begin{array}{c}\text { Thickness } \\
(\mathbf{m m})\end{array}$ & LLRF \\
\hline Story6 & P1 & 6000 & 4500 & 14000 & 250 & 0.774 \\
\hline
\end{tabular}

Table 9 shows the material properties.

Table 9: Material properties

\begin{tabular}{c|c|c|c|c}
\hline $\mathbf{E}_{c}(\mathbf{M P a})$ & $\mathbf{f}_{\mathrm{ck}}(\mathbf{M P a})$ & $\begin{array}{c}\text { Lt.Wt Factor } \\
\text { (Unitless) }\end{array}$ & $\mathbf{f}_{\mathbf{s}}(\mathbf{M P a})$ & $\mathbf{f}_{\mathrm{ys}}(\mathbf{M P a})$ \\
\hline 27386.13 & $\beta 0$ & 1 & 413.69 & 413.69 \\
\hline
\end{tabular}

Table 10 shows the design code parameters.

Table 10: Design code parameters

\begin{tabular}{c|c|c|c|c|c|c}
\hline $\boldsymbol{\Gamma}_{\mathrm{S}}$ & $\boldsymbol{\Gamma}_{\mathrm{C}}$ & $\mathbf{I P}_{\mathrm{MAX}}$ & $\mathbf{I P}_{\mathbf{M I N}}$ & $\mathbf{P}_{\mathrm{MAX}}$ & $\begin{array}{c}\text { MinEcc } \\
\text { Major }\end{array}$ & $\begin{array}{c}\text { MinEcc } \\
\text { Minor }\end{array}$ \\
\hline 1.15 & 1.5 & 0.04 & 0.0025 & 0.8 & Yes & Yes \\
\hline
\end{tabular}

Table 11 shows the Pier Leg Locations, Length and Thickness

Table 11: Pier Leg Locations, Length and Thickness

\begin{tabular}{c|c|c|c|c|c|c|c}
\hline $\begin{array}{c}\text { Station } \\
\text { Location }\end{array}$ & ID & $\begin{array}{c}\text { Left } \mathbf{X}_{\mathbf{1}} \\
\mathbf{m m}\end{array}$ & $\begin{array}{c}\text { Left } \mathbf{Y}_{\mathbf{1}} \\
\mathbf{m m}\end{array}$ & $\begin{array}{c}\text { Right } \mathbf{X}_{\mathbf{2}} \\
\mathbf{m m}\end{array}$ & $\begin{array}{c}\text { Right } \mathbf{Y}_{\mathbf{2}} \\
\mathbf{m m}\end{array}$ & $\begin{array}{c}\text { Length } \\
\mathbf{m m}\end{array}$ & $\begin{array}{c}\text { Thickness } \\
\mathbf{m m}\end{array}$ \\
\hline Top & Leg 1 & 4000 & 3000 & 8000 & 3000 & 4000 & 250 \\
\hline Top & Leg 2 & 8000 & 3000 & 8000 & 6000 & 3000 & 250 \\
\hline Top & Leg 3 & 4000 & 6000 & 8000 & 6000 & 4000 & 250 \\
\hline Top & Leg 4 & 4000 & 3000 & 4000 & 6000 & 3000 & 250 \\
\hline Bottom & Leg 1 & 4000 & 6000 & 8000 & 6000 & 4000 & 250 \\
\hline Bottom & Leg 2 & 8000 & 3000 & 8000 & 6000 & 3000 & 250 \\
\hline Bottom & Leg 3 & 4000 & 3000 & 8000 & 3000 & 4000 & 250 \\
\hline Bottom & Leg 4 & 4000 & 3000 & 4000 & 6000 & 3000 & 250 \\
\hline
\end{tabular}

Table 12 shows the Flexural Designs for $\mathrm{P}_{\mathrm{u}}, \mathrm{M}_{\mathrm{u} 2}$ and $\mathrm{M}_{\mathrm{u} 3}$

Table 12: Flexural Designs for $P_{u}, M_{u 2}$ and $M_{u 3}$

\begin{tabular}{|c|c|c|c|c|c|c|c|c|}
\hline $\begin{array}{c}\text { Station } \\
\text { Locatio } \\
\mathbf{n}\end{array}$ & $\begin{array}{c}\text { Required } \\
\text { Rebar } \\
\text { Area } \\
\left(\mathrm{mm}^{2}\right)\end{array}$ & \begin{tabular}{|c|} 
Required \\
Reinf Ratio
\end{tabular} & $\begin{array}{l}\text { Current } \\
\text { Reinf } \\
\text { Ratio }\end{array}$ & $\begin{array}{l}\text { Fexural } \\
\text { Combo }\end{array}$ & $\begin{array}{l}\mathrm{P}_{4} \\
\mathrm{bN}\end{array}$ & $\begin{array}{c}\mathbf{M}_{\mathrm{nz}} \\
\mathrm{LNN}-\mathrm{m}\end{array}$ & $\begin{array}{c}\mathbf{M}_{25} \\
\mathrm{kN}-\mathrm{m}\end{array}$ & $\begin{array}{c}\text { Fier } A_{2} \\
\mathrm{~mm}^{2}\end{array}$ \\
\hline Top & 8750 & 0.0025 & 0.0087 & DWals & 228.182 & 8.2526 & 0 & 3500000 \\
\hline Bottom & 8750 & 0.0025 & 0.0087 & DWals & 503.7256 & -24.463 & $\begin{array}{c}- \\
279.4445\end{array}$ & 35000 \\
\hline
\end{tabular}

Table 13 shows the shear design.

Table 13: Shear Design

\begin{tabular}{|c|c|c|c|c|c|c|c|c|}
\hline \begin{tabular}{c|} 
Station \\
Location
\end{tabular} & ID & \begin{tabular}{|c|} 
Rebar \\
$\mathrm{mm}^{2} / \mathrm{m}$
\end{tabular} & $\begin{array}{l}\text { Shear } \\
\text { Combo }\end{array}$ & $\begin{array}{l}P_{4} \\
W N\end{array}$ & $\begin{array}{c}\mathbf{M}_{\mathbf{a}} \\
\mathrm{WN}-\mathrm{m}\end{array}$ & $\begin{array}{l}V_{a} \\
b N\end{array}$ & $\begin{array}{l}\mathrm{X}_{\mathrm{C}} \\
\mathrm{WN}\end{array}$ & $\begin{array}{c}\mathrm{X}_{\mathrm{c}}+\mathrm{V}_{\mathrm{u}} \\
\mathrm{bN}\end{array}$ \\
\hline Top & $\operatorname{Leg} 1$ & 625 & DWal5 & 08.6581 & 60.8281 & 8365 & 4520 & 539739 \\
\hline Top & $\operatorname{Leg} 2$ & 625 & Wal6 & 81.4936 & 183.0154 & -91.0722 & 175.8907 & 715.4804 \\
\hline Top & eg 3 & 625 & DWal5 & 52.7573 & 60.8281 & 128.3651 & 233.224 & 952.677 \\
\hline Top & eg 4 & 625 & Wal6 & 69.0495 & 183.0154 & 91.0722 & 175.6019 & 715.1917 \\
\hline Bottom & Leg 1 & 625 & Wal5 & 339686 & 210.4249 & 28365 & 236.2681 & 955.7211 \\
\hline Bottom & Leg 2 & 625 & DWal6 & 179902 & 313757 & -91.0722 & 178.1737 & 717.7635 \\
\hline Bottom & eg 3 & 625 & DWal5 & 239.8693 & 210.4249 & 128.36 & 237565 & 957.018 \\
\hline Botto & 24 & 625 & Val6 & 7.4579 & 31.3757 & 0722 & 77.885 & 7.4748 \\
\hline
\end{tabular}

Table 14 shows the boundary element check.

Table 14: Boundary Element Check

\begin{tabular}{|c|c|c|c|c|c|c|c|}
\hline $\begin{array}{c}\text { Station } \\
\text { Location }\end{array}$ & ID & \begin{tabular}{|c} 
Edge \\
Leugth \\
(mm)
\end{tabular} & $\begin{array}{c}\text { Governing } \\
\text { Combo }\end{array}$ & $\begin{array}{c}\mathrm{P}_{4} \\
\mathrm{kN}\end{array}$ & $\underset{\mathrm{WN}-\mathrm{m}}{\mathrm{M}_{2}}$ & $\begin{array}{l}\text { Stress } \\
\text { Comp } \\
\text { MPR }\end{array}$ & $\begin{array}{l}\text { Stress } \\
\text { Limit } \\
\text { MPR }\end{array}$ \\
\hline Top-Left & $\operatorname{Leg} 1$ & 0 & DWals & 1645589 & -238.853 & 0.52 & 6 \\
\hline Top-Right & Leg 1 & 0 & DWal5 & 1645589 & 60.8281 & 0.26 & 6 \\
\hline Top-Left & Leg2 & 0 & DWal6 & 939377 & -32.7026 & 0.21 & 6 \\
\hline Top-Right & Leg 2 & 0 & DWal6 & 93.9377 & 183.0154 & 0.61 & 6 \\
\hline Top-Left & Leg 3 & 0 & DWal6 & 1645589 & -60.8281 & 0.26 & 6 \\
\hline Top-Right & Leg 3 & 0 & DWal6 & 1645589 & 238.853 & 0.52 & 6 \\
\hline Top-Left & Leg 4 & 0 & DWal5 & 939377 & -183.0154 & 0.61 & 6 \\
\hline Top-Right & $\operatorname{Leg} 4$ & 0 & DWal5 & 939377 & 32.7026 & 0.21 & 6 \\
\hline $\begin{array}{c}\text { Bottom- } \\
\text { Left }\end{array}$ & Leg 1 & 0 & DWal6 & 295.7701 & -210.4249 & 0.61 & 6 \\
\hline $\begin{array}{c}\text { Bottom- } \\
\text { Right }\end{array}$ & Leg 1 & 0 & DWal6 & 295.7701 & 63.0265 & 0.39 & 6 \\
\hline $\begin{array}{c}\text { Bottom- } \\
\text { Left }\end{array}$ & Leg 2 & 0 & DWal6 & 192.3461 & -135.7371 & 0.62 & 6 \\
\hline $\begin{array}{c}\text { Bottom- } \\
\text { Right }\end{array}$ & Leg 2 & 0 & DWal6 & 192.3461 & 31.3757 & 0.34 & 6 \\
\hline $\begin{array}{c}\text { Bottom- } \\
\text { Left } \\
\end{array}$ & Leg 3 & 0 & DWal5 & 295.7701 & -63.0265 & 0.39 & 6 \\
\hline $\begin{array}{c}\text { Bottom- } \\
\text { Right }\end{array}$ & Leg 3 & 0 & DWal5 & 295.7701 & 210.4249 & 0.61 & 6 \\
\hline $\begin{array}{c}\text { Bottom- } \\
\text { Left }\end{array}$ & $\operatorname{Leg} 4$ & 0 & DWal5 & 192.3461 & $-31375 ?$ & 0.34 & 6 \\
\hline $\begin{array}{c}\text { Bottom- } \\
\text { Right }\end{array}$ & $\operatorname{Leg} 4$ & 0 & DWal5 & 192.3461 & 135.7371 & 0.62 & 6 \\
\hline
\end{tabular}

\section{Conclusion}

From our project we conclude that shear wall is highly safe when ii is experimentally and analytically tested and implemented. It generated results are compared and concluded for maximum optimum level of output.

\section{References}

[1] T.Subramani, J.Jayalakshmi, " Analytical Investigation Of Bonded Glass Fibre Reinforced Polymer Sheets With Reinforced Concrete Beam Using Ansys" , International Journal of Application or Innovation in Engineering \& Management (IJAIEM) , Volume 4, Issue 5, pp. 105-112, 2015

[2] T.Subramani. , S.Vishnupriya, "Finite Element Analysis of a Natural Fiber (Maize) Composite Beam", International Journal of Modern Engineering Research, Volume. 4, Issue. 6 (Version 1), pp $1-7,2014$,

[3] T.Subramani., R.Senthil Kumar, "Modelling and Analysis of Hybrid Composite Joint Using Fem in ANSYS", International Journal of Modern Engineering Research, Volume 4, Issue 6 (Version 1), pp 41- 46, 2014.

[4] T.Subramani, S.Sharmila, "Prediction of Deflection and Stresses of Laminated Composite Plate with Artificial Neural Network Aid", International Journal of Modern Engineering Research, Volume 4, Issue 6 (Version 1), pp $51-58,2014$.

[5] T.Subramani., S.Sundar, M.Senthilkumar, "Investigation of the Behaviour for Reinforced Concrete Beam Using Non Linear Three Dimensional Finite Elements", International Journal of Modern Engineering Research, Volume. 4, Issue. 6 (Version 2), pp 13 -18, 2014,

[6] T.Subramani, A.Arul, "Design And Analysis Of Hybrid Composite Lap Joint Using Fem" International Journal of Engineering 
Research and Applications, Volume. 4, Issue. 6 (Version 5), pp 289- 295, 2014.

[7] T.Subramani., J.Jothi,, M.Kavitha "Earthquake Analysis Of Structure By Base Isolation Technique In SAP", International Journal of Engineering Research and Applications, Volume. 4 Issue. 6 (Version 5), pp 296 - 305, 2014.

[8] T.Subramani., R.Manivannan.R, M.Kavitha, "Crack Identification In Reinforced Concrete Beams Using Ansys Software" ,International Journal of Engineering Research and Applications, Volume. 4, Issue. 6 (Version 6), pp 133 - 141, 2014.

[9] T.Subramani., Reni Kuruvilla, J.Jayalakshmi., "Nonlinear Analysis Of Reinforced Concrete Column With Fiber Reinforced Polymer Bars" International Journal of Engineering Research and Applications Volume. 4, Issue. 6 (Version 5), pp 306- 316, 2014.

[10] T.Subramani, D.Sakthi Kumar, S.Badrinarayanan. "Fem Modelling And Analysis Of Reinforced Concrete Section With Light Weight Blocks Infill " International Journal of Engineering Research and Applications, Volume. 4, Issue. 6 (Version 6), pp 142 - 149, 2014.

[11] T.Subramani, B.Saravanan., J.Jayalakshmi., "Dynamic Analysis Of Flanged Shear Wall Using Staad Pro", International Journal of Engineering Research and Applications, Volume. 4, Issue. 6 (Version 6), pp 150 - 155, 2014.

[12] T.Subramani, M.Subramani., K.Prasath.,"Analysis Of Three Dimensional Horizontal Reinforced Concrete Curved Beam Using Ansys" International Journal of Engineering Research and Applications, Volume. 4, Issue. 6 (Version 6), pp 156 - 161, 2014.

[13] T.Subramani., K.Bharathi Devi., M.S.Saravanan. , Suboth Analysis Of RC Structures Subject To Vibration By Using Ansys," International Journal of Engineering Research and Applications Vol. 4, Issue 12(Version 5), pp.45-54, 2014.

[14] T.Subramani., K.Bharathi Devi., M.S.Saravanan., Suboth Thomas, "Analysis Of Seismic Performance Of Rock Block Structures With STAAD Pro International Journal of Engineering Research and Applications Vol. 4, Issue 12(Version 5), pp.55- 68, 2014

[15] T.Subramani., T.Krishnan., M.S.Saravanan.M , Suboth Thomas, "Finite Element Modeling On Behaviour Of Reinforced Concrete Beam Column Joints Retrofitted With CFRP Sheets Using Ansys" International Journal of Engineering Research and Applications Vol. 4, Issue 12(Version 5), pp.69 -76, 2014

[16] T.Subramani., S.Krishnan., M.S.Saravanan.M, Suboth Thomas "Analysis Of Retrofitting Non-Linear Finite Element Of RCC Beam And Column Using Ansys" International Journal of Engineering Research and Applications ,Vol. 4, Issue 12(Version 5), pp.77-87, 2014

[17] T.Subramani, J.Jayalakshmi , " Analytical Investigation Of Bonded Glass Fibre Reinforced Polymer Sheets With Reinforced Concrete Beam Using Ansys" , International Journal of Application or Innovation in Engineering \& Management (IJAIEM) , Volume 4, Issue 5, pp. 105-112, 2015

[18] T.Subramani and M.Kavitha, "Analysis Of Reliability Of Steel Frame Systems With Semi-Rigid Connections Using Numerical Method And Finite Element Analysis", International Journal of Applied Engineering Research (IJAER), Volume 10, Number 38,Special Issues, pp.28240-28246, 2015

[19] T.Subramani, M.S.Saravanan, “Analysis Of Non Linear Reinforced And Post Tensioned Concrete Beams Using ANSYS", International Journal of Applied Engineering Research (IJAER) International Journal of Applied Engineering Research (IJAER), Volume 10, Number 38 Special Issues, pp.28247-28252, 2015

[20] T.Subramani, K.Balamurugan , " Finite Element Anaylsis Of Composite Element For FRP Reinforced Concrete Slab By Using ANSYS" , International Journal of Application or Innovation in Engineering \& Management (IJAIEM), Volume 5, Issue 5, pp. 076-084, 2016

[21] T.Subramani, V.Kanian Poonkundran , " Prefabricated Multistory Structure Exposure To Engineering Seismicity By Using SAP" , International Journal of Application or Innovation in Engineering \& Management (IJAIEM) , Volume 5, Issue 5, pp. 123-131 , 2016.

[22] T.Subramani, A.Kumaravel , " Analysis Of Polymer Fibre Reinforced Concrete Pavements By Using ANSYS" , International Journal of Application or Innovation in Engineering \& Management (IJAIEM), Volume 5, Issue 5, pp. 132-139, 2016 T.Subramani, R.Praburaj , " Pushover Anaylsis Of Retrofitted Reinforced Concrete Buildings By Using SAP" , International Journal of Application or Innovation in Engineering \& Management (IJAIEM), Volume 5, Issue 5, pp. 140-147, 2016.

[24] T.Subramani, M.Senthilkumar, " Finite Element Anaylsis Of RC Beams With Externally Bonded Simcon Laminates By Using
ANSYS" , International Journal of Application or Innovation in Engineering \& Management (IJAIEM), Volume 5, Issue 5, pp. $148-155,2016$

[25] T.Subramani, R.Vasanthi, " Earth Quake Resistant Building Using SAP" , International Journal of Application or Innovation in Engineering \& Management (IJAIEM), Volume 5, Issue 5, pp. $173-181,2016$.

[26] T.Subramani, A.Selvam , "Studies On Economical Configuration Of RCC And Prestressed Shell Roofs By Using ANSYS " , International Journal of Application or Innovation in Engineering \& Management (IJAIEM), Volume 5, Issue 5, pp. 182-191, 2016. T.Subramani, A.Anbuchezian," Experimental Investigation On Flexural Behavior Of Folded Ferro Cement Panels " , International Journal of Application or Innovation in Engineering \& Management (IJAIEM), Volume 6, Issue 3, March 2017 , pp. 045049 , ISSN 2319 - 4847.

[28] T.Subramani, A. Fizoor Rahman, " An Experimental Study On The Properties Of Pet Fibre Reinforced Concrete " , International Journal of Application or Innovation in Engineering \& Management (IJAIEM), Volume 6, Issue 3, March 2017 , pp. 058066 , ISSN 2319 - 4847.

[29] T.Subramani, S.Poongothai, S.Priyanka , " Analytical Study Of T Beam Column Joint Using FEM Software " , International Journal of Emerging Trends \& Technology in Computer Science (IJETTCS), Volume 6, Issue 3, May - June 2017, pp. 148-156 , ISSN 2278-6856

[30] T.Subramani, R.Ganapathy,V.Manoharan, M.Balamurugan, R.Murugesan, " Design And Analysis Of Light Weight Concrete Building Using ETAB With Respect To Dynamic Loading " , International Journal of Emerging Trends \& Technology in Computer Science (IJETTCS), Volume 6, Issue 3, May - June 2017 , pp. 252-258, ISSN 2278-6856.

[31] T.Subramani, A.Mohammed Ali, R.Karthikeyan, E.Panner Selvan, K.Periyasamy , " Analytical Study Of T-Beam Using ANSYS " , International Journal of Emerging Trends \& Technology in Computer Science (IJETTCS), Volume 6, Issue 3, May - June 2017 , pp. 259-266, ISSN 2278-6856.

[32] T.Subramani, V.Kalaivanan, S.Priyaranjithkumar, P.Sasikumar, P.Vinoth Kumar, " Design And Analysis Of Multistorey Building With Respect To Seismic Loads Using ETABS " , International Journal of Emerging Trends \& Technology in Computer Science (IJETTCS), Volume 6, Issue 3, May - June 2017, pp. 267-274, ISSN 2278-6856. 